\title{
BMJ Open Gaps in antihypertensive and statin treatments and benefits of optimisation: a modelling study in a 1 million ethnically diverse urban population in UK
}

\author{
Runguo Wu (D) ,' Stuart Christopher Gorthorn Rison (D) ,,2 \\ Zahra Raisi-Estabragh, ${ }^{3,4}$ Isabel Dostal, ${ }^{1}$ Chris Carvalho (D) , ${ }^{1,5}$ John Robson, ${ }^{1}$ \\ Borislava Mihaylova (i) ${ }^{1,6}$
}

To cite: Wu R, Rison SCG, Raisi-Estabragh Z, et al. Gaps in antihypertensive and statin treatments and benefits of optimisation: a modelling study in a 1 million ethnically diverse urban population in UK. BMJ Open 2021;0:e052884. doi:10.1136/ bmjopen-2021-052884

- Prepublication history and additional supplemental material for this paper are available online. To view these files, please visit the journal online (http://dx.doi.org/10.1136/ bmjopen-2021-052884).

Received 27 April 2021 Accepted 02 December 2021

Check for updates

(C) Author(s) (or their employer(s)) 2021. Re-use permitted under CC BY. Published by BMJ.

For numbered affiliations see end of article.

Correspondence to Professor Borislava Mihaylova; b.mihaylova@qmul.ac.uk

\section{ABSTRACT}

Objectives To characterise gaps in antihypertensive treatment in people with hypertension and statin treatment in people with cardiovascular diseases (CVD) in a large urban population and quantify the health and economic impacts of their optimisation. Design A cross-sectional population study and a longterm CVD decision model.

Setting Primary care, UK.

Participants All adults with diagnosed hypertension or CVD in a population of about 1 million people, served by 123 primary care practices in London, UK in 2019.

Interventions Following UK clinical guidelines, all adults with diagnosed hypertension were categorised into optimal, suboptimal and untreated groups with respect to their antihypertensive treatment, and all adults with diagnosed CVD were categorised in the same manner with respect to their statin treatment.

Outcomes Proportion of patients suboptimally treated or untreated. Projected cardiovascular events avoided, years and quality-adjusted life years (QALYs) gained and healthcare costs saved with optimised treatments.

Results 21954 of the 91828 adults with hypertension (24\%; mean age 59 years; $49 \%$ women) and 9062 of the 23723 adults with CVD (38\%; mean age 69 years; $43 \%$ women) were not optimally treated with antihypertensive or statin treatment, respectively. Per 1000 additional patients optimised over 5 years, hypertension treatment is projected to prevent 25 (95\% Cl 16 to 32) major vascular events (MVEs) and 7 ( 3 to 10) vascular deaths, statin treatment, 28 (22 to 33) MVEs and 6 (4 to 7) vascular deaths. Over their lifespan, a patient with uncontrolled hypertension aged $60-69$ years is projected to gain 0.64 ( $95 \% \mathrm{Cl} 0.36$ to 0.87 ) QALYs with optimised hypertension treatment, and a similarly aged patient with previous CVD not optimally treated with statin is projected to gain 0.3 (0.24 to 0.37 ) QALYs with optimised statin treatment. In both cases, the hospital cost savings minus extra medication costs were about $£ 1100$ per person over remaining lifespan.

Conclusions Optimising cardiovascular treatments can cost-effectively reduce cardiovascular risk and improve life expectancy.
Strengths and limitations of this study

- This study quantifies the gaps in blood pressure and statin treatments among people at high cardiovascular disease risk in a large ethnically diverse UK urban population.

- A cardiovascular disease model projects net health outcomes and extra healthcare costs with treatment optimisation to guide prioritisation of efforts.

- The study does not assess specific interventions to improve uptake and adherence to recommended treatments.

\section{INTRODUCTION}

Cardiovascular disease (CVD) is the most common cause of morbidity and mortality worldwide, and hypertension and hypercholesterolaemia are two of its key modifiable risk factors. ${ }^{1}$ Their widespread suboptimal treatment, however, represents a substantial missed opportunity for CVD prevention. ${ }^{2}$ Reducing raised systolic blood pressure by $10 \mathrm{~mm} \mathrm{Hg}$ with antihypertensive treatment decreases risks of ischaemic heart disease (IHD) and stroke by $25 \%-35 \%,{ }^{3}{ }^{4}$ and reducing low-density lipoprotein cholesterol (LDL-C) by $1 \mathrm{mmol} / \mathrm{L}$ with statin therapy reduces these risks by $24 \%-25 \%$ with more intensive statin regimens achieving larger risk reductions. ${ }^{5}$ In the UK, the National Institute for Health and Care Excellence (NICE) recommends antihypertensive medications for people with high blood pressure ${ }^{6}$ and high-intensity statin treatment for people with CVD. ${ }^{7}$ However, gaps in treatment initiation and poor patient adherence to treatment are common ${ }^{89}$ and a strategy to highlight the benefits and complexity of treating to targets, 
and to structure routine practice to facilitate medicines optimisation, has been put forward. ${ }^{10}$

East London is a geographic area in London, UK with an ethnically diverse urban population. More than $70 \%$ of east London areas are categorised in the bottom two quintiles of country's socioeconomic deprivation. ${ }^{11}$ Primary care practices in east London are looking to implement a programme to optimise blood pressure and lipid control to improve quality of CVD management and reduce health inequality in the population. In this study, we describe the gaps in antihypertensive and statin treatments in east London and project the health and healthcare costs with their optimisation.

\section{METHODS}

\section{Study population}

Data were extracted for all adult patients (aged $\geq 18$ years on 1 January 2020) with diagnosis of hypertension or CVD among a population of about 1 million people registered with all 123 primary care practices across three Clinical Commissioning Groups (CCGs) in east London (City and Hackney, Newham and Tower Hamlets). The local authorities covered by these NHS services are among the $10 \%$ most socially deprived areas in England, and the ethnically diverse population includes large South Asian and Black British, African and Caribbean ethnic groups. ${ }^{11}$

The review of blood pressure treatment included patients with diagnosed hypertension, and the review of cholesterol-lowering treatment included patients with diagnosed ischaemic CVD (myocardial infarction, angina and other IHD, peripheral artery disease, any stroke or transient ischaemic attack). The extracted data included primary care practice code, individual's age, sex, ethnicity, Index of Multiple Deprivation (IMD) ${ }^{11}$ quintile, smoking status, measures of total and high-density lipoprotein cholesterol (HDL-C), triglycerides, creatinine, systolic and diastolic blood pressure, prescribed cholesterollowering and antihypertensive medications, previous CVD, hypertension, diabetes and chronic kidney disease (CKD). Individual LDL-C levels were calculated using the Friedewald formula. ${ }^{12}$

For a small number of patients, data were missing for IMD, sex, ethnicity, smoking status, cholesterol, creatinine and blood pressure (6.5\% for HDL-C, $<5 \%$ for others). Missing IMD quintiles $(0.05 \%)$ were assigned to the most populous IMD quintile by primary care practice. Missing ethnicities $(2.5 \%)$ were assigned to the most common ethnic group for the area. Missing sex (one patient) was assigned to 'men', reflecting greater proportion of men in the study population. Other missing values were imputed using multiple imputation with chained equations, including all patient characteristics as covariates. ${ }^{13}$

\section{The Heart Protection Study CVD policy model}

The Heart Protection Study-CVD (HPS-CVD) policy model, ${ }^{14}$ a Markov model employing parametric survival models for CVD endpoints and a linear regression model for annual hospital care costs, developed using the individual participant data of the HPS, was used to project the outcomes in the present study without and with treatment optimisation. The non-vascular mortality rates in the model, originally based on lifetable data for England, was replaced with 2019 mortality data for the three east London CCGs (online supplemental table S1). The original cardiovascular risk equations were adjusted for ethnicity (by sex) in patients without previous CVD (online supplemental table S2) ${ }^{15}$ and calibrated to year 2018 using decreasing CVD trends in England between 2001 and 2018 (online supplemental table S3) ${ }^{1617}$ Based on patient characteristics at entry, the model projects annual risks of vascular and non-vascular death, nonfatal major vascular event (MVE: myocardial infarction, stroke, arterial revascularisation) and other vascular event (admission for angina, heart failure or other cardiac or vascular problem).

\section{Antihypertensive treatment}

Optimising antihypertensive treatment was considered for all patients diagnosed with hypertension whose latest blood pressure measurement indicated suboptimal control (ie, systolic/diastolic blood pressure $>140 / 90$ $\mathrm{mm} \mathrm{Hg}$ for those aged $<80$ years, or $>150 / 90 \mathrm{~mm} \mathrm{Hg}$ for those aged $\geq 80$ years, according to NICE guidance ${ }^{6}$ ), irrespective of measurement setting ( $>99 \%$ of blood pressure measures were made in clinic). Blood pressure treatment, including up to three antihypertensive agents, was categorised in line with NICE guidance. ${ }^{6}$

The antihypertensive medications were grouped into angiotensin converting enzyme inhibitors (ACEi)/ angiotensin receptor blockers (ARB), calcium channel blockers (CCB), thiazide diuretics (TD) and others (including beta blockers, spironolactone/potassiumsparing diuretics, alpha blockers and loop diuretic). In the optimisation strategy, first-line treatment recommended by NICE was applied for patients who were not using any antihypertensive treatment. TD was added for patients already on the recommended first-line treatment; ACEi/ARB or CCB, respectively, was added (as per indicated first-line treatment) to those on antihypertensive medication different from the recommended first-line recommendation; ACEi/ARB, CCB or TD, respectively, was added for patients already on the other two categories of antihypertensives. No further optimisation was considered for patients who already used a combination of ACEi/ARB, CCB and TD treatment independently of their achieved blood pressure; these patients are regarded as having resistant hypertension ${ }^{6}$ and beyond the scope of this optimisation programme.

Ramipril $5 \mathrm{mg}$ /day was used for ACEi/ARB treatment, amlodipine $5 \mathrm{mg}$ /day for CCB treatment and indapamide $2.5 \mathrm{mg} /$ day for TD treatment with additive effects with use of two or more categories. ${ }^{18}$ These regimens were expected to achieve systolic blood pressure reductions of 7.47 (95\% CI 2.19 to 12.76$),{ }^{19} 8.9$ (7.66 to 10.14$)^{20}$ and $11.94(7.99 \text { to } 15.88)^{21} \mathrm{~mm} \mathrm{Hg}$, respectively. A tenth of 
the difference between preoptimised systolic blood pressure values $(\mathrm{mm} \mathrm{Hg})$ and a reference value $(154 \mathrm{~mm} \mathrm{Hg})$ was deducted from (preoptimised systolic blood pressure $<154$ ) or added to (preoptimised systolic blood pressure $>154$ ) the expected systolic blood pressure reduction in line with the finding that greater reduction is elicited when treating a higher initial blood pressure (on average, a further $1 \mathrm{~mm} \mathrm{Hg}$ systolic blood pressure reduction per $10 \mathrm{~mm} \mathrm{Hg}$ preoptimised systolic blood pressure above $154 \mathrm{~mm} \mathrm{Hg}) .{ }^{18}$ The effects of antihypertensive treatments were estimated using the expected systolic blood pressure reductions and the HRs for reductions in cardiovascular events per $\mathrm{mm} \mathrm{Hg}$ systolic blood pressure reduction with antihypertensive treatment, reported by the Blood Pressure Lowering Treatment Trialists' Collaboration (online supplemental table S4). ${ }^{34}$

\section{Cholesterol-lowering treatment with statins}

Statin treatment optimisation was considered for patients with history of CVD not receiving statin treatment or receiving suboptimal low or medium-intensity treatment. For such patients, the current NICE guidance recommends starting statin treatment with atorvastatin $80 \mathrm{mg}$, unless the patient has $\mathrm{CKD}$, there is high risk of adverse effects or alternative preference; the recommended statin treatment is irrespective of starting LDL-C concentration. ${ }^{7}$ To assess the achieved reduction in LDL-C under ongoing treatments, the cholesterol-lowering regimens used by patients were grouped into three intensity levels with expected LDL-C reduction ${ }^{7}$ of $\geq 45 \%$ for highintensity, $35 \%-45 \%$ for medium-intensity and $<35 \%$ for low-intensity regimens (see online supplemental table S5 for more details).

The optimisation strategy followed the NICE guidance and used atorvastatin $80 \mathrm{mg}$ in patients aged under 75 years and without CKD, and atorvastatin $40 \mathrm{mg}$ in patients aged 75 years and older or with CKD (online supplemental table S6). ${ }^{22}$ The effects of statin treatments were projected using effects of statin therapy per $1 \mathrm{mmol} / \mathrm{L}$ LDL-C reduction, reported by Cholesterol Treatment Trialists' (CTT) Collaboration meta-analysis of randomised studies (online supplemental table S4). ${ }^{5}$ The effect of statin regimens used prior to optimisation was simulated based on achieved reductions in LDL-C at 1 year in randomised controlled trials included in CTT in categories by low/medium or high-intensity regimens. ${ }^{5}$ Expected further reduction in LDL-C achieved with the optimised statin therapy was determined by the preoptimised LDL-C level and the difference between proportional reductions in LDL-C achieved by the optimised and preoptimised statin regimens (online supplemental table S7).

\section{Healthcare costs and health-related quality of life}

Annual hospital care costs in the HPS model were inflated to 2019 (online supplemental table S8). ${ }^{23}$ The extra medication costs were estimated as the difference between the cost of the optimised treatment and the costs of the respective preoptimisation treatments (online supplemental table S9). ${ }^{24}$

We estimated health-related quality of life (QoL) related to patient characteristics, including experience of cardiovascular events, using a linear regression model of EuroQol-5 dimension utility related to individual characteristics, using data from the Health Surveys for England (online supplemental table S10). The QoL utility values range from -0.594 for the worst health state to 1 for full health, where 0 is a health state equivalent to death and higher values indicate better QoL. ${ }^{25}$ The patients' predicted QoL during each year in the model was combined with their predicted survival to estimate quality-adjusted life year (QALY).

\section{Effects of optimisation strategies}

The model projected non-fatal MVEs, other non-fatal vascular events, deaths from vascular causes, survival (ie, life years), QALYs, annual hospital care costs and additional medication costs. The added life years and QALYs gained were calculated for three optimisation scenarios: optimising $10 \%$ and $20 \%$ additional patients from respective overall target populations and optimising all patients not on optimal treatment. The results are presented by age category $(<50,50-59,60-69,70-79$ and $\geq 80$ years $)$, with projections over 5 years, 10 years and over patients' lifespans. The parameter uncertainty was assessed using the non-parametric bootstrap approach and 1000 nonparametric bootstrap resamples in HPS model. ${ }^{14}$ The uncertainty in risk ratios of statin and antihypertensive treatments used sampling from their respective lognormal distributions.

While antihypertensive and statin treatments were optimised separately in the two study populations, in a scenario analysis, the effect of optimising both antihypertensive and statin treatments in patients with hypertension and CVD and not on optimal antihypertensive nor optimal statin treatment was evaluated.

The analytical framework is presented in figure 1. All analyses were performed in R 4.0.2.

\section{RESULTS}

\section{Baseline characteristics of the study population}

There were 91828 patients diagnosed with hypertension and 23723 patients with prior CVD in the three east London CCGs. The study population had high levels of deprivation, with $93 \%$ of individuals in the bottom two quintiles of the socioeconomic deprivation in England and was highly ethnically diverse ( $35 \%$ white, $31 \%$ South Asian and 26\% black). Of the patients with diagnosed hypertension, 21954 (24\%) were not on optimal antihypertensive treatment, and in 2867 (3\%) patients, the treatment status was unknown due to missing blood pressure measures (table 1). Of patients not on optimal treatment, 18 $282(83 \%)$ were on antihypertensive treatment, but their blood pressure was not at target and they were 


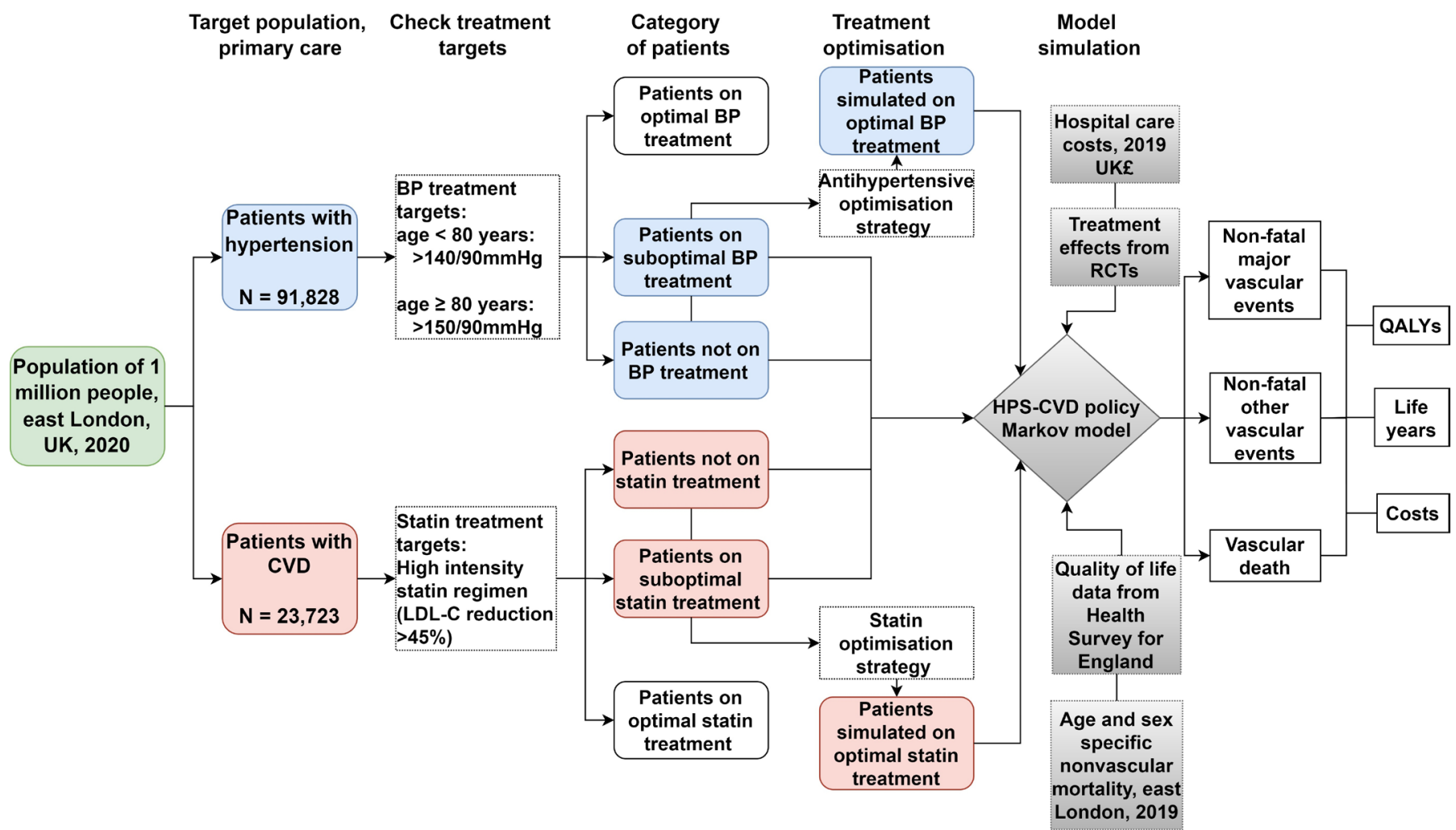

Figure 1 A flowchart of the procedure of the data analysis and model projection. CVD, cardiovascular disease; BP, blood pressure; LDL-C, low-density lipoprotein cholesterol; HPS, Heart Protection Study; QALY, quality-adjusted life year; RCT, randomised controlled trial.

not on triple antihypertensive treatment with combination of ACEi/ARB, CCB and TD, and 3672 (17\%) were not treated with any antihypertensive medication (table 1 and online supplemental tables S11,12). Of the patients with prior CVD, $9062(38 \%)$ were not on optimal cholesterol-lowering treatment (table 1), of whom, $5729(63 \%)$ were on suboptimal low or medium-intensity cholesterol-lowering treatment, and $3333(37 \%)$ were untreated (table 1, online supplemental tables S5,S12).

Among patients with diagnosed hypertension, men were less likely to be on optimal antihypertensive treatment compared with women $(74 \%$ vs $76 \%$; $\mathrm{p}<0.001)$, as were patients of black $(72 \%)$ compared with white $(76 \% ; \mathrm{p}<0.001)$ or South Asian $(77 \%$; $\mathrm{p}<0.001$ ) ethnicities (table 1). Among patients with previous CVD, women were less likely to be on optimal cholesterol-lowering treatment compared with men ( $56 \%$ vs $65 \% ; \mathrm{p}<0.001)$, as were patients of black ethnicity $(57 \%)$ compared with white $(60 \%$; $\mathrm{p}<0.01)$ or South Asian $(67 \%$; $\mathrm{p}<0.001)$ ethnicities. Patients with diagnosed hypertension not on optimal antihypertensive treatment were younger (mean age 59 vs 64 years; $p<0.001$ ), while patients with previous CVD not on optimal cholesterol-lowering treatment were older ( 69 vs 66 years; $p<0.001)$. Compared with all other socioeconomic quintiles together, patients in the most deprived quintile were more likely to be on optimal antihypertensive $(76 \%$ vs $75 \%$; $<<0.01)$ and cholesterol-lowering (64\% vs $59 \% ; \mathrm{p}<0.001)$ treatment.

\section{Projected health benefits with optimised treatments}

Optimising treatments was projected to substantially reduce CVD risks (table 2). Optimising antihypertensive treatment in 1000 patients with hypertension was evaluated to lead to 25 (95\% CI 16 to 32) fewer non-fatal MVEs and 7 ( 3 to 10) fewer vascular deaths in 5 years, and 151 (72 to 223) fewer non-fatal MVEs and 65 (27 to 98) fewer vascular deaths over patients' lifetimes. Similarly, the optimisation of statin treatment in 1000 patients with CVD was projected to lead to 28 (22 to 33) fewer non-fatal MVEs and 6 (4 to 7) fewer vascular deaths in 5 years, and 139 (100 to 173) fewer non-fatal MVEs and 31 (22 to 38) fewer vascular deaths over patient lifetimes.

Optimising antihypertensive and, separately, statin treatments was predicted to importantly improve life expectancy and QALYs over patient lifetimes (table 3). The predicted QALY gains from optimised antihypertensive treatment in patients with hypertension ranged from 1.11 (95\% CI 0.64 to 1.49$)$ QALYs for those aged under 50 years to 0.31 ( 0.17 to 0.43$)$ QALYs for those aged 80 years or over, with 0.24 (0.12 to 0.33) MVEs and 0.05 (0.00 to 0.09) MVEs avoided, respectively (online supplemental table S13). The estimated QALY gains for patients with CVD from optimised statin treatment ranged from 0.72 (0.55 to 0.90) QALY for those aged under 50 years to 0.12 (0.09 to 0.14) QALYs for those aged 80 years or over, with 


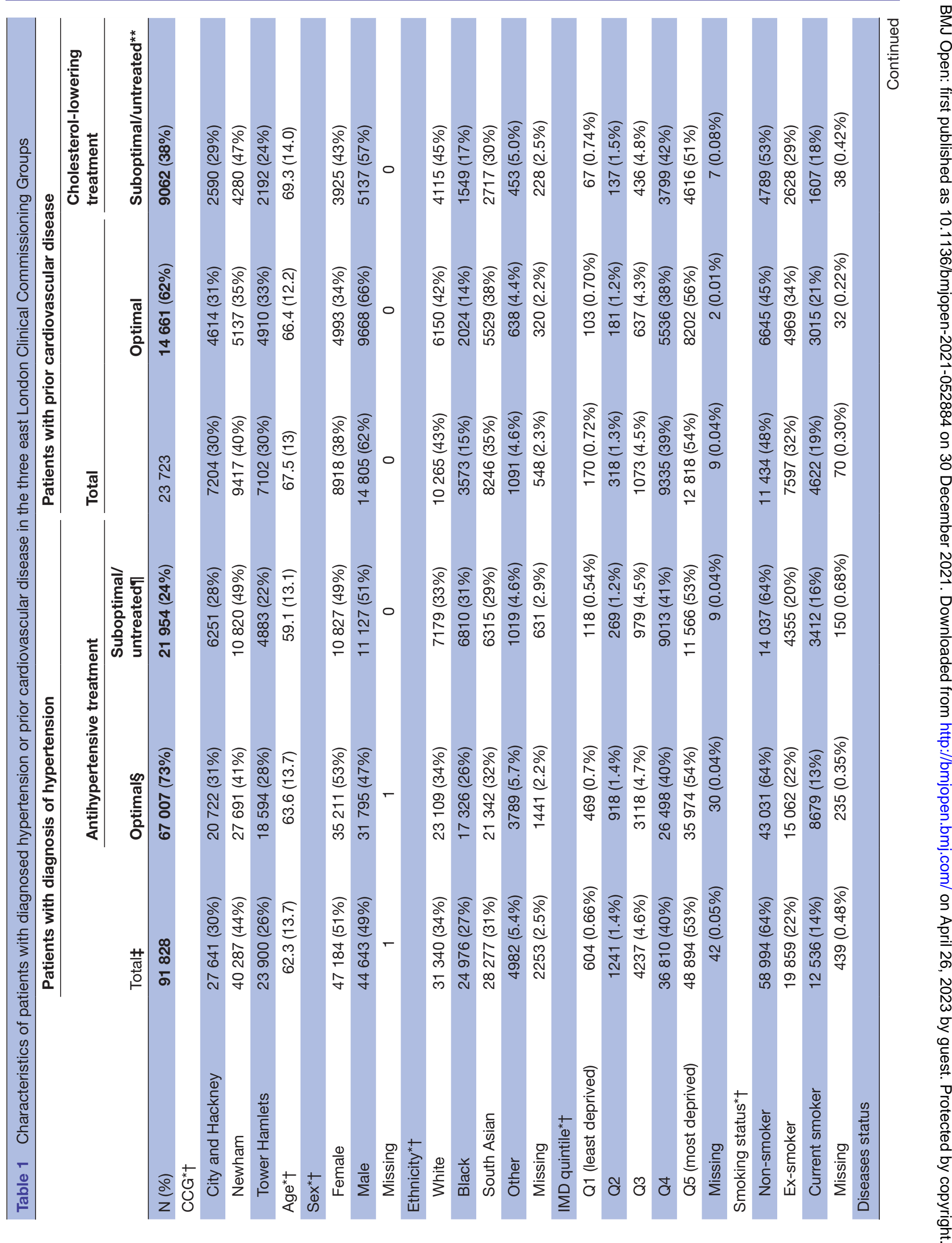




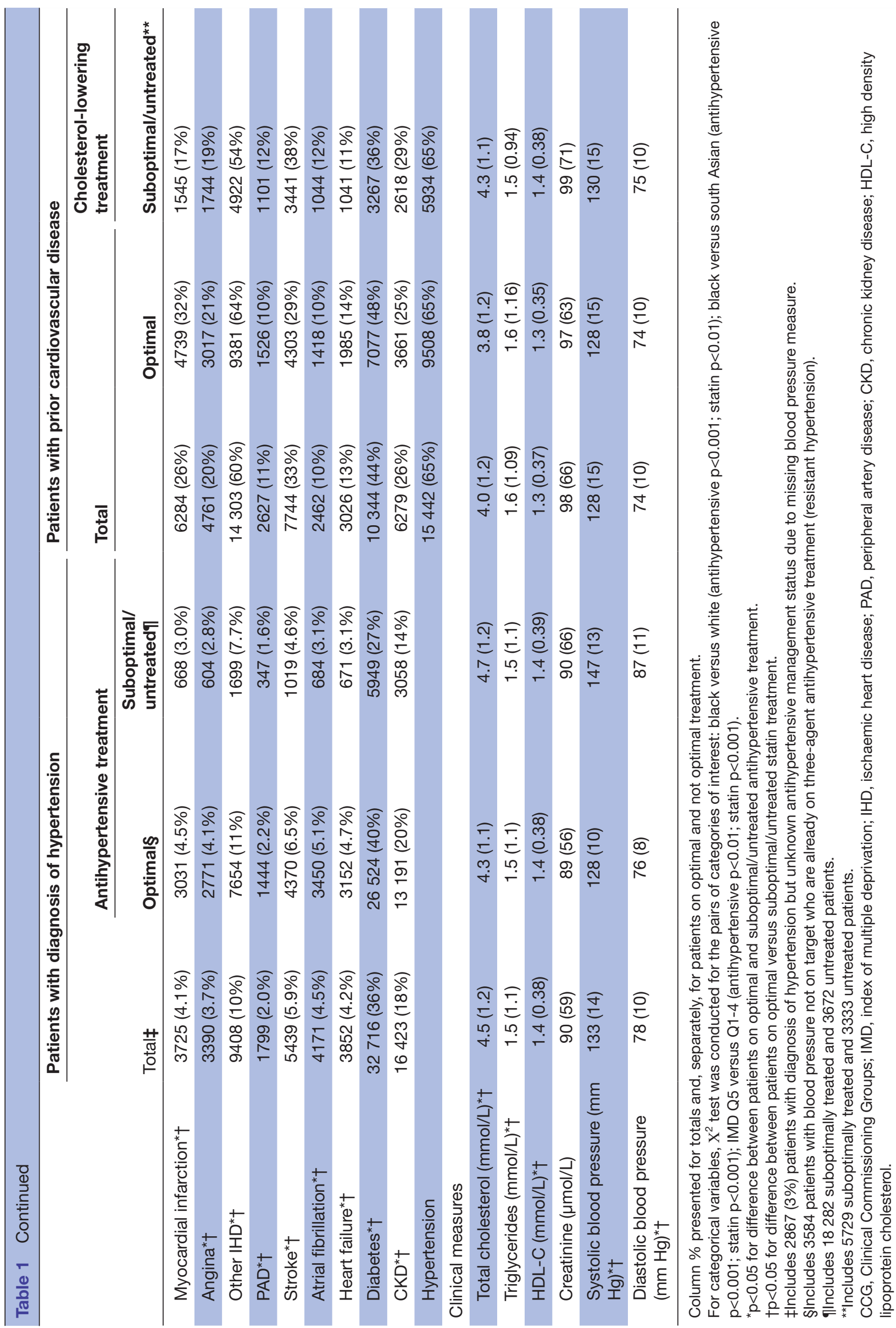

งิ

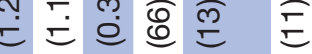

艺

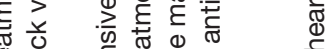

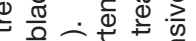
ब

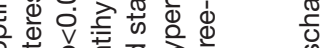

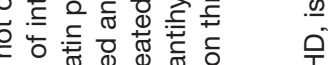

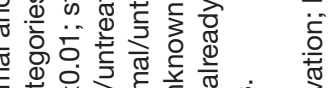

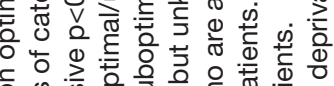

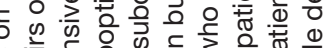

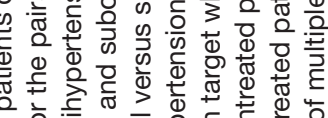

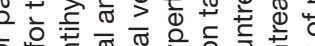

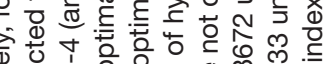
б б ᄃ ᄃ

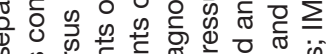

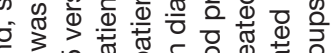

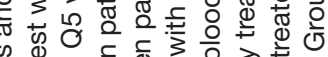
$\frac{\infty}{\bar{\sigma}}+$ क ष

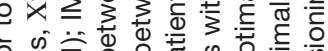


Table 2 Predicted reductions in cardiovascular events with optimised antihypertensive or statin treatment

Cardiovascular events avoided with optimised antihypertensive or statin treatment

Vascular deaths

Non-fatal MVEs avoided (per 1000 Non-fatal OVEs avoided (per avoided (per 1000 treated) $(95 \% \mathrm{Cl})$ 1000 treated) $(95 \% \mathrm{Cl})$ treated) $(95 \% \mathrm{Cl})$

Time horizon

Patients with hypertension not on optimal antihypertensive treatment $(\mathrm{N}=22191)^{*}$

\begin{tabular}{|c|c|c|c|}
\hline 5 years & 25 (16 to 32 ) & 32 (24 to 40 ) & 7 (3 to 10) \\
\hline 10 years & 50 (31 to 67) & 67 (49 to 83) & 15 (6 to 22) \\
\hline Lifetime & 151 (72 to 223 ) & 209 (140 to 274$)$ & 65 (27 to 98) \\
\hline 5 years & 28 (22 to 33) & 15 (4 to 26$)$ & 6 (4 to 7$)$ \\
\hline 10 years & 54 (42 to 65) & 29 (8 to 50) & 12 (9 to 15$)$ \\
\hline
\end{tabular}

Only one vascular event is simulated during each year in the model with priority given to more severe events.

MVE, major vascular events, defined as non-fatal myocardial infarction or death from coronary disease, any stroke, or revascularisation procedure. OVE, other vascular event, defined as admission for angina, heart failure, or other cardiac or vascular problem.

*237 patients with hypertension and unknown blood pressure were categorised into this group following missing blood pressure multiple imputation.

CVD, cardiovascular disease.

0.34 (0.25 to 0.42$)$ and 0.05 (0.04 to 0.07$)$ MVEs avoided, respectively (online supplemental table S13). Optimising antihypertensive and statin treatments was also predicted to reduce hospital care costs (table 3 ). Both health benefits and cost savings were larger among younger patients.

\section{Projected programme impacts}

Optimisation of antihypertensive treatment in all patients with hypertension in the three east London CCGs was predicted to lead to 22228 (95\% CI 9234 to 33 296) more life years, 16698 (9485 to 22 482) more QALYs, and to save $£ 41069942$ (6 894396 to 74751579 ) in hospital care costs (online supplemental table S14, figure S1). Similarly, optimisation of statin treatment in all patients with prior CVD in the three east London CCGs was predicted to lead to 4034 (2948 to 5059) more life years, 2616 (2022 to 3184) more QALYs, and to save $£ 11603287$ (7 243739 to 15597438 ) in hospital care costs.

The benefits from optimising suboptimal antihypertensive treatments were larger than those from optimising those not on antihypertensive treatment, while the opposite was true with optimising statin treatment, where larger benefits were projected among patients not on cholesterol-lowering treatment (figure 2, online supplemental table S15). Optimising both antihypertensive and statin treatments in patients needing both optimised, resulted in complementary gains in life years, QALYs and hospital care cost saving (online supplemental table S16).

\section{Scenario analyses}

Given that $73 \%$ of the patients with diagnosed hypertension were on optimal antihypertensive treatment before treatment optimisation, a $10 \%$ increase in $83 \%$ required 9183 extra patients to be optimised. Over lifetime, this scenario was projected to achieve 1393 fewer non-fatal MVEs and 601 fewer vascular deaths, respectively, and to add 9220 life years and 6925 QALYs, respectively (online supplemental figure S1). A $10 \%$ increase in the proportion of the patients with CVD on optimal statin treatment (ie, from $62 \%$ to $72 \%$ ) required treatment optimisation in 2372 additional patients. Over lifetime, this scenario was estimated to lead to 326 fewer non-fatal MVEs and 72 fewer vascular deaths and increase life years by 1044 and QALYs by 683, respectively (online supplemental figure S1). The benefits were doubled by a $20 \%$ increase in the optimised patients (online supplemental figure S1).

\section{DISCUSSION}

This analysis of primary care records of a large inner city population in London, UK indicates that $27 \%$ of patients with hypertension and $38 \%$ of patients with CVD do not receive optimal antihypertensive and cholesterollowering treatments, respectively. The subsequent projections, using a CVD model, suggest that optimising the use of these treatments will achieve substantial further reductions in cardiovascular events and gains in survival and QoL while also reducing hospital care costs with larger benefits achieved among younger patients. Moreover, given the concentration of hypertension and CVD among people in the highest categories of socioeconomic deprivation, and the disproportionately high percentage of suboptimal treatment among patients of black ethnicity, the optimisation of cardiovascular prevention treatments will also reduce health inequalities.

Despite study population situated among local authorities in the highest socioeconomic deprivation decile in England, ${ }^{11}$ the proportions of optimally managed patients in our study are high, both compared with other local authorities in England, ${ }^{26}$ and to European and worldwide data ${ }^{927}$ and were not adversely associated 
Table 3 Predicted lifetime gains in survival and QALYs, hospital care cost savings, and additional medication cost of fully optimised antihypertensive and statin treatment

\begin{tabular}{|c|c|c|}
\hline & $\begin{array}{l}\text { Patients with hypertension not on } \\
\text { optimal antihypertensive treatment }\end{array}$ & $\begin{array}{l}\text { Patients with prior CVD not on optimal } \\
\text { statin treatment }\end{array}$ \\
\hline Age (years) & $\mathrm{N}=22191$ & $\mathrm{~N}=9062$ \\
\hline \multicolumn{3}{|c|}{ Life years gained per optimised patient $(95 \% \mathrm{Cl})$} \\
\hline$<50$ & $1.36(0.57$ to 2.04$)$ & 1.03 (0.74 to 1.32$)$ \\
\hline $50-59$ & $1.08(0.45$ to 1.61$)$ & $0.72(0.52$ to 0.91$)$ \\
\hline $60-69$ & 0.91 (0.38 to 1.36$)$ & 0.47 (0.34 to 0.59$)$ \\
\hline $70-79$ & 0.66 (0.27 to 0.98$)$ & 0.30 (0.22 to 0.37$)$ \\
\hline$\geq 80$ & $0.54(0.22$ to 0.80$)$ & $0.22(0.16$ to 0.27$)$ \\
\hline \multicolumn{3}{|c|}{ QALYs gained per optimised patient $(95 \% \mathrm{Cl})$} \\
\hline$<50$ & $1.11(0.64$ to 1.49$)$ & $0.72(0.55$ to 0.9$)$ \\
\hline $50-59$ & $0.83(0.47$ to 1.12$)$ & 0.49 (0.37 to 0.59$)$ \\
\hline $60-69$ & 0.64 (0.36 to 0.87$)$ & 0.30 (0.24 to 0.37$)$ \\
\hline $70-79$ & $0.43(0.24$ to 0.59$)$ & $0.18(0.14$ to 0.22$)$ \\
\hline$\geq 80$ & 0.31 (0.17 to 0.43$)$ & 0.12 (0.09 to 0.14$)$ \\
\hline \multicolumn{3}{|c|}{ Hospital care cost savings (£) per optimised patient $(95 \% \mathrm{Cl})$} \\
\hline$<50$ & 3100 (1040 to 5092$)$ & 3508 (2432 to 4472 ) \\
\hline $50-59$ & 2058 (417 to 3653$)$ & 2272 (1494 to 2978) \\
\hline $60-69$ & 1448 (36 to 2879 ) & 1373 (862 to 1841$)$ \\
\hline $70-79$ & $831(-224$ to 1910$)$ & 751 (414 to 1082$)$ \\
\hline$\geq 80$ & $353(-552$ to 1242$)$ & 390 (146 to 629$)$ \\
\hline \multicolumn{3}{|c|}{ Extra medication costs $(£)$ per optimised patient $(95 \% \mathrm{Cl})$} \\
\hline$<50$ & 712 (692 to 728$)$ & 728 (712 to 744$)$ \\
\hline $50-59$ & 521 (507 to 533) & 406 (395 to 416 ) \\
\hline $60-69$ & 389 (377 to 399$)$ & 248 (241 to 254$)$ \\
\hline $70-79$ & 267 (258 to 274$)$ & 114 (110 to 117$)$ \\
\hline$\geq 80$ & 159 (152 to 165$)$ & 54 (52 to 56 ) \\
\hline
\end{tabular}

1203 patients with hypertension and previous CVD included in both patient categories.

CVD, cardiovascular disease; QALY, quality-adjusted life year.

with level of deprivation. This has been achieved over 20 years of locally sustained, digitally supported and incentivised quality improvement programmes. ${ }^{28}$ However, our data suggest that there is still scope for improvement in management of blood pressure and cholesterol, and the improved understanding of where the gaps are will inform strategic choices. In the present study, patients not on optimal antihypertensive treatment, most without previous CVD, were more likely to be younger and men, while those not optimally treated with statin, in a population with previous CVD, were older and women. Patients of black ethnicity were also more likely to require treatment optimisation. Similar demographic and ethnic associations with suboptimal use of cardiovascular prevention medications have been previously reported ${ }^{929}$ and point to a need for action.

The benefits from optimising treatments to reduce CVD accrue over patient lifetimes and vary across categories of patients. Differences in characteristics of patients requiring optimisation lead to different sizes of benefits from optimisation, which, in our study, are larger in younger and predominantly without previous CVD population requiring optimisation of blood pressure, compared with the population with history of CVD requiring statin treatment optimisation. Despite variation in size of benefits, however, successful treatment optimisation is shown to be highly beneficial across all patient categories studied.

Our study indicates that there are differences between suboptimally treated and untreated individuals in terms of mean age, comorbidities and other risk factors; these differences also vary between the two treatments studied. Among individuals with increased blood pressure, optimising suboptimal treatment resulted in larger benefits compared with optimising untreated patients because of higher disease risks in this category, and also because effects of antihypertensive treatments are additive. ${ }^{18}$ The opposite was the case among people with CVD, where 


\section{A Predicted reductions in cardiovascular events and gains in life years and QALYs from antihypertensive treatment optimisation for patients with hypertension (per 1000 treated)}

Non-fatal MVE avoided

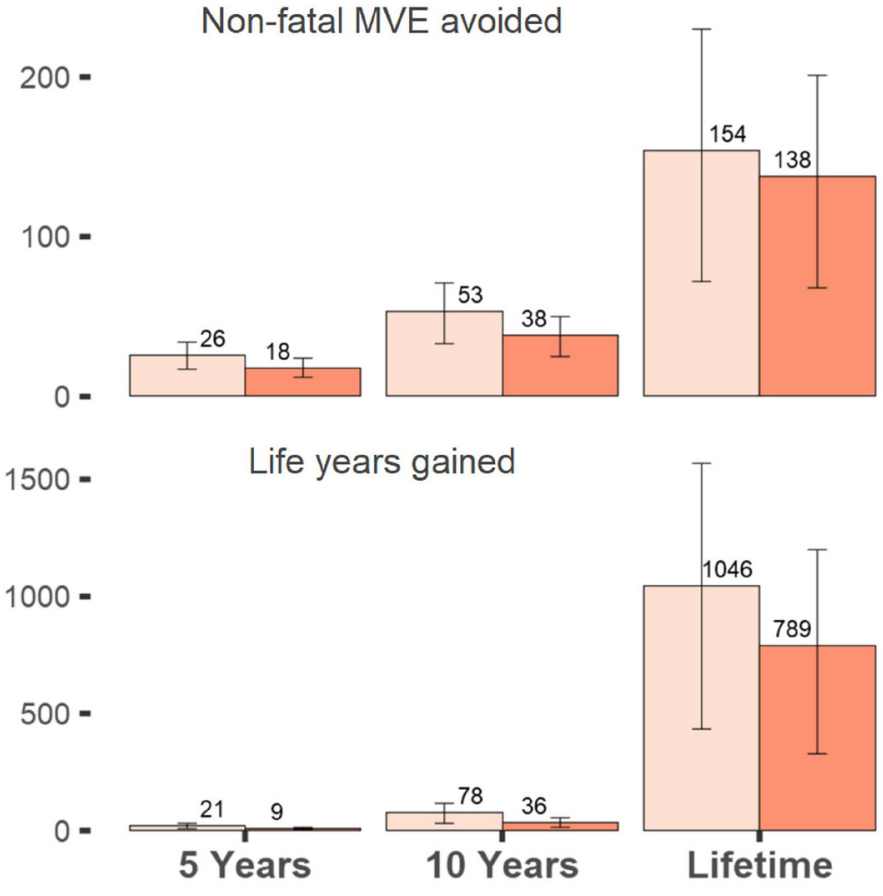

Vascular death avoided

Suboptimal to optimal

No treatment to optimal

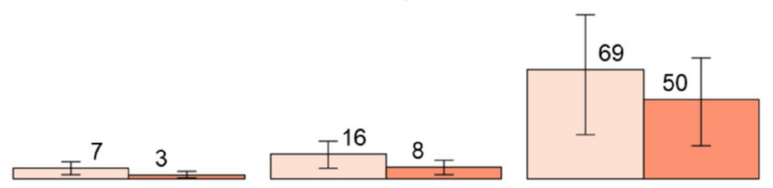

QALYs gained
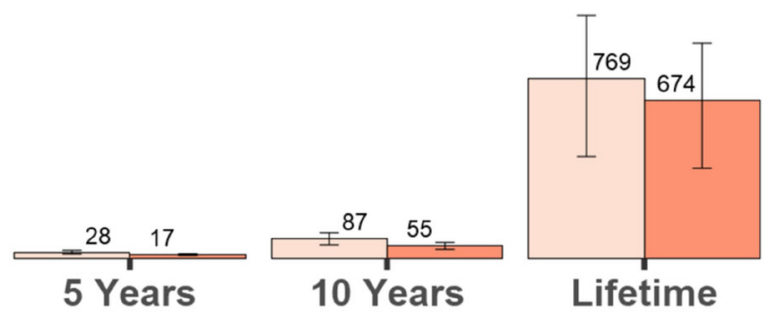

Suboptimal to optimal

No treatment to optimal
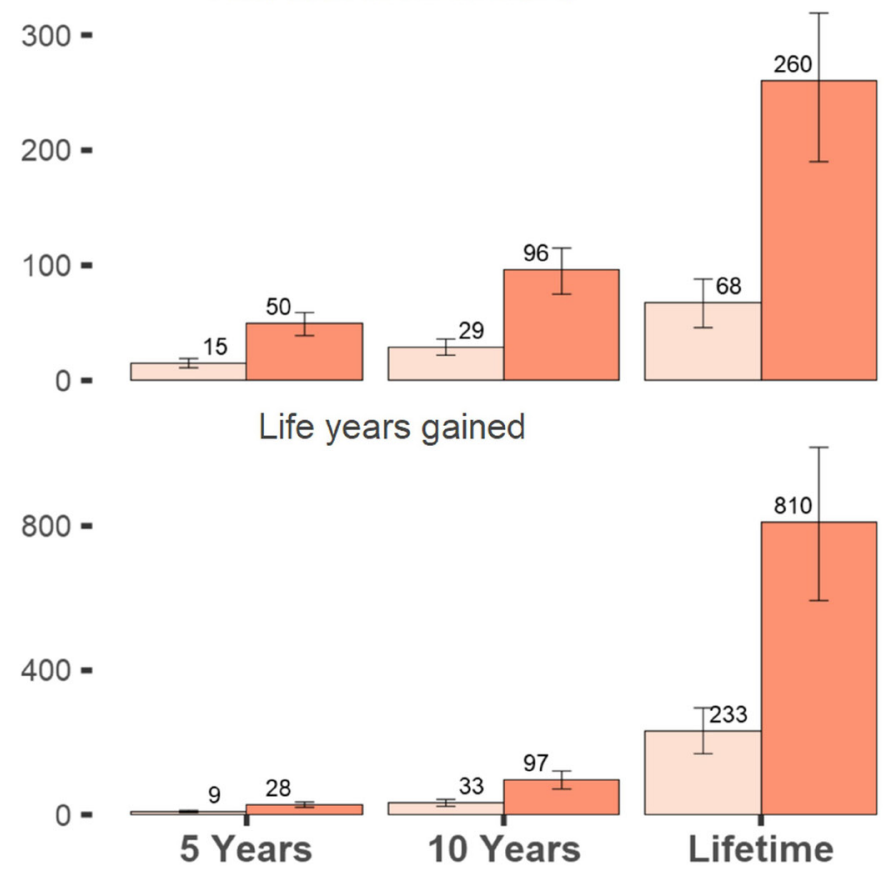

$800-$

$400-$

B Predicted reductions in cardiovascular events and gains in life years and QALYs from statin treatment optimisation for patients with prior CVD (per 1000 treated)

Non-fatal MVE avoided

Vascular death avoided

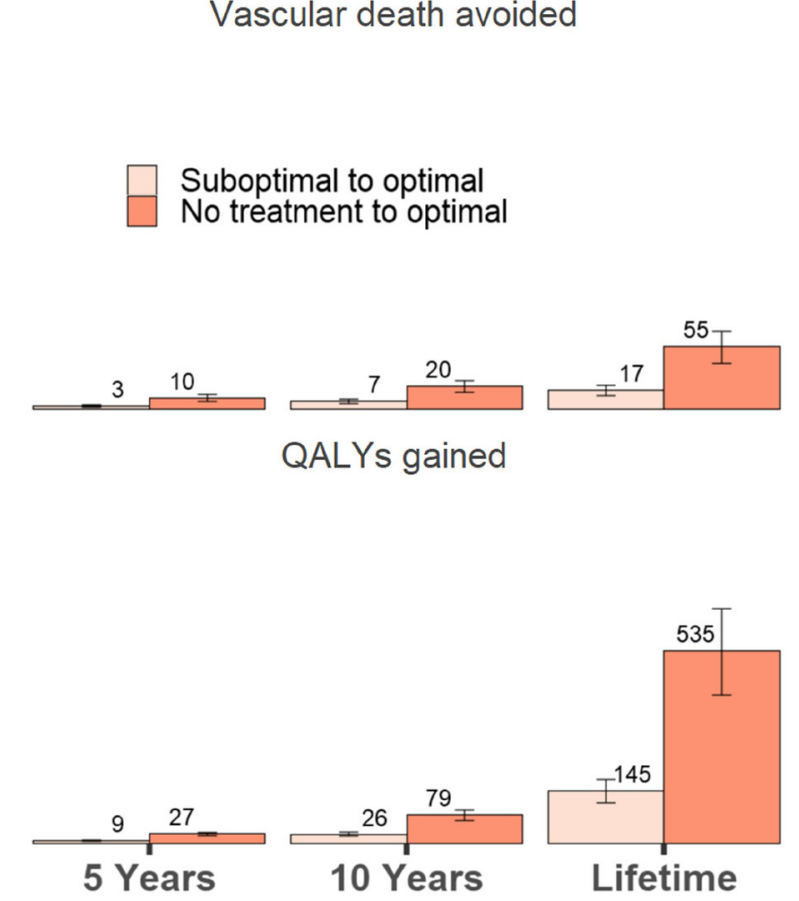

Figure 2 Model projected benefits from antihypertensive and statin treatment optimisation for patients previously on suboptimal treatment or not treated. CVD, cardiovascular disease; MVE, major vascular event; QALY, quality-adjusted life year.

benefits from optimising statin treatment were much larger among untreated compared with suboptimally treated, mainly because increasing treatment intensity, for example, doubling of statin dose, produces only $6 \%$ reduction in LDL cholesterol. ${ }^{30}$ This study falls short, however, of evaluating cost-effectiveness of programmes to optimise cardiovascular prevention in primary care. Such evaluations require estimates of programme's impact on uptake and adherence to optimal treatment as well as the cost of programme implementation and management. 
Interventions to improve adherence to statin and blood pressure treatments have been an area of active research, though with limited success so far. ${ }^{31}{ }^{32}$ Complex interventions, combining elements targeting knowledge and behaviour of patients and clinicians and facilitation from the health system, have shown some promise, and the projections reported in the present study can facilitate the evaluation of cost-effectiveness of such programmes.

Our study has some further limitations. First, while we adapted and calibrated the HPS-CVD model for use in the present study, these adjustments were based on published estimates and population statistics for England rather than data specific to the study population. Second, we present results under full adherence to optimal treatments to motivate action; suboptimal adherence, however, may erode the benefits projected here. Third, we did not account for adverse effects of antihypertensive and statin treatments alone or in combination with concomitant medications, which may result in drug withdrawal and reductions in QALYs. Fourth, while we report the cost for additional blood pressure and statin medications, we did not account for any additional consultations and testing in primary care to initiate and support people on treatment. There is, however, ongoing effort in UK primary care to optimise monitoring and management across individual's needs and, therefore, extra consultations may not be needed. ${ }^{33}$ Finally, this study only included patients with hypertension and previous CVD and focused on bloodpressure lowering and statin treatments; future investigations could be extended to optimising statin treatment for primary CVD prevention or indeed consider the use of novel interventions, such as PCSK9 inhibitors, to further reduce population CVD risks. ${ }^{34}$

In conclusion, our paper presents a comprehensive population study in 1 million people in east London, an urban area with high ethnic diversity and high deprivation. We report gaps in antihypertensive treatment among patients with hypertension and in statin treatment among patients with previous CVD. Optimising these treatments will reduce vascular events, increase life expectancy and QALYs and reduce hospital care costs while also reducing socioeconomic inequalities in health. Developments of programmes to bring forward improvements in initiation and adherence to cardiovascular preventive interventions need to be prioritised.

\section{Author affiliations}

${ }^{1}$ Wolfson Institute of Population Health, Barts and the London School of Medicine and Dentistry, Queen Mary University of London, London, UK

${ }^{2}$ Bromley-by-Bow Health Centre, London, UK

${ }^{3}$ William Harvey Research Institute, NIHR Barts Biomedical Research Centre, Queen

Mary University of London, London, UK

${ }^{4}$ Barts Heart Centre, St Bartholomew's Hospital, Barts Health NHS Trust, London, UK ${ }^{5}$ De Beauvoir Surgery, London, UK

${ }^{6}$ Nuffield Department of Population Health, University of Oxford, Oxford, UK

\section{Twitter Stuart Christopher Gorthorn Rison @CEG_Twt}

Contributors JR and BM conceived the study. All authors contributed to study design and development. JR, SCGR, ZR-E and CC advised on the treatment optimisation protocol. JR, SCGR and ID contributed to acquisition of data. RW and
BM performed the data analyses and wrote the first draft of the manuscript. All authors provided critical comments on the interpretation of the results and the revision of manuscript and approved the final version. BM is responsible for the overall content and is a guarantor.

Funding This study was supported by the Barts Charity REAL-Health Cardiovascular disease (reference GPPG1J4R). ZR-E was supported by British Heart Foundation Clinical Research Training Fellowship (reference FS/17/81/33318). JR is supported by and contributing to Health Data Research UK funded by the UK Medical Research Council, Engineering and Physical Sciences Research Council, Economic and Social Research Council, Department of Health and Social Care (England), Chief Scientist Office of the Scottish Government Health and Social Care Directorates, Health and Social Care Research and Development Division (Welsh Government), Public Health Agency (Northern Ireland), British Heart Foundation and Wellcome. (reference GPPB1C2). The funders took no role in the analysis, interpretation and writing of this report.

Competing interests None declared.

Patient consent for publication Not applicable.

Ethics approval Ethical approval was not required as patient-level data are anonymised, and only aggregated patient data are reported in this study. All GPs in the participating east London practices consented to the use of their anonymised patient data for research and development for patient benefit.

Provenance and peer review Not commissioned; externally peer reviewed.

Data availability statement All data relevant to the study are included in the article or uploaded as supplementary information. All general practitioners in the participating east London practices consented to the use of their anonymised patient data for research and development for patient benefit.

Supplemental material This content has been supplied by the author(s). It has not been vetted by BMJ Publishing Group Limited (BMJ) and may not have been peer-reviewed. Any opinions or recommendations discussed are solely those of the author(s) and are not endorsed by BMJ. BMJ disclaims all liability and responsibility arising from any reliance placed on the content. Where the content includes any translated material, BMJ does not warrant the accuracy and reliability of the translations (including but not limited to local regulations, clinical guidelines, terminology, drug names and drug dosages), and is not responsible for any error and/or omissions arising from translation and adaptation or otherwise.

Open access This is an open access article distributed in accordance with the Creative Commons Attribution 4.0 Unported (CC BY 4.0) license, which permits others to copy, redistribute, remix, transform and build upon this work for any purpose, provided the original work is properly cited, a link to the licence is given, and indication of whether changes were made. See: https://creativecommons.org/ licenses/by/4.0/.

\section{ORCID iDs}

Runguo Wu http://orcid.org/0000-0002-6523-5754

Stuart Christopher Gorthorn Rison http://orcid.org/0000-0003-3289-6668

Chris Carvalho http://orcid.org/0000-0002-4114-9210

Borislava Mihaylova http://orcid.org/0000-0002-0951-1304

\section{REFERENCES}

1 NICE. Cardiovascular disease: risk assessment and reduction, including lipid modification (clinical guideline CG181). London: National Institute for Health and Care Excellence, 2014. https://www. nice.org.uk/guidance/cg181/chapter/Introduction

2 Sheppard JP, Fletcher K, McManus RJ, et al. Missed opportunities in prevention of cardiovascular disease in primary care: a crosssectional study. Br J Gen Pract 2014;64:e38-46.

3 Blood Pressure Lowering Treatment Trialists' Collaboration. Effects of different blood-pressure-lowering regimens on major cardiovascular events: results of prospectively-designed overviews of randomised trials. Lancet 2003;362:1527-35.

4 Blood Pressure Lowering Treatment Trialists' Collaboration. Blood pressure lowering and major cardiovascular events in people with and without chronic kidney disease: meta-analysis of randomised controlled trials. BMJ 2013;347:f5680.

5 Cholesterol Treatment Trialists' (CTT) Collaboration. Efficacy and safety of more intensive lowering of LDL cholesterol: a meta-analysis of data from 170,000 participants in 26 randomised trials. Lancet 2010;376:1670-81. 
6 NICE. Hypertension in adults: diagnosis and management (NICE guideline NG136). London: National Institute for Health and Care Excellence, 2019. https://www.nice.org.uk/guidance/ng136/chapter/ Recommendations\#starting-antihypertensive-drug-treatment

7 NICE. Cardiovascular disease: risk assessment and reduction, including lipid modification (clinical guideline CG181). London: National Institute for Health and Care Excellence, 2014. https://www. nice.org.uk/guidance/cg181/chapter/1-Recommendations\#lipidmodification-therapy-for-the-primary-and-secondary-prevention-ofcvd-2 accessed 16/09/2020

8 Pinho-Gomes AC, Peters SAE, Thomson B, et al. Sex differences in prevalence, treatment and control of cardiovascular risk factors in England. Heart 2020;107:462-7.

9 Achelrod D, Gray A, Preiss D, et al. Cholesterol- and blood-pressurelowering drug use for secondary cardiovascular prevention in 20042013 Europe. Eur J Prev Cardiol 2017;24:426-36.

10 Phillips LS, Branch WT, Cook CB, et al. Clinical inertia. Ann Intern Med 2001;135:825-34.

11 UK Government. English indices of deprivation 2015. Ministry of Housing, Communities \& Local Government, 2015.

12 Friedewald WT, Levy RI, Fredrickson DS. Estimation of the concentration of low-density lipoprotein cholesterol in plasma, without use of the preparative ultracentrifuge. Clin Chem 1972;18:499-502.

13 Buuren SV, Groothuis-Oudshoorn K. Mice: multivariate imputation by chained equations in R. J Statist Software 2010:1-68.

14 Heart Protection Study Collaborative. Lifetime cost effectiveness of simvastatin in a range of risk groups and age groups derived from a randomised trial of 20536 people. BMJ 2006;333:1145.

15 Hippisley-Cox J, Coupland C, Brindle P. Development and validation of QRISK3 risk prediction algorithms to estimate future risk of cardiovascular disease: prospective cohort study. BMJ 2017;357:j2099.

16 Office for National Statistics. Mortality statistics - underlying cause, sex and age, 2019. https://www.ons.gov.uk/peoplepopulationandc ommunity/birthsdeathsandmarriages/deaths/bulletins/deathsregist rationsummarytables/2019

17 Cegedim SA. The health improvement network (THIN), 2020.

18 Law MR, Wald NJ, Morris JK, et al. Value of low dose combination treatment with blood pressure lowering drugs: analysis of 354 randomised trials. BMJ 2003;326:1427.

19 Heran BS, Wong MMY, Heran IK, et al. Blood pressure lowering efficacy of angiotensin converting enzyme (ACE) inhibitors for primary hypertension. Cochrane Database Syst Rev 2008;78.

20 Wright JM, Musini VM, Gill R. First-line drugs for hypertension. Cochrane Database Syst Rev 2018;4:CD001841.
21 Musini VM, Nazer M, Bassett K. Blood pressure-lowering efficacy of monotherapy with thiazide diuretics for primary hypertension. Cochrane Database Syst Rev 2014;5.

22 Clinical Effectiveness Group. Statin guidance update London: Barts and the London School of Medicine and Dentistry. Queen Mary University of London, 2015. https://www.towerhamletsccg.nhs.uk/ CEG\%20Statin\%20update\%202015.pdf

23 Curtis L, Burns A. Unit costs of health and social care 2019. Personal Social Services Research Unit, University of Kent: Canterbury, 2019.

24 NHS Business Services Authority. Prescription cost analysis England 2019. Newcastle upon Tyne, 2020.

25 Dolan P. Modeling valuations for EuroQol health states. Med Care 1997;35:1095-108.

26 Steel N, Ford JA, Newton JN, et al. Changes in health in the countries of the UK and 150 English local authority areas 1990-2016: a systematic analysis for the global burden of disease study 2016 . Lancet 2018;392:1647-61.

27 Chow CK, Teo KK, Rangarajan S, et al. Prevalence, awareness, treatment, and control of hypertension in rural and urban communities in high-, middle-, and low-income countries. JAMA 2013;310:959-68.

28 Robson J, Boomla K, Hull SA. Progress in using the electronic health record to improve primary care. Br J Gen Pract 2020;70:e215-20.

29 De Smedt D, De Backer T, Petrovic M, et al. Chronic medication intake in patients with stable coronary heart disease across Europe: evidence from the daily clinical practice. results from the ESC EORP European survey of cardiovascular disease prevention and diabetes (EUROASPIRE IV) registry. Int J Cardiol 2020;300:7-13.

30 Collins R, Reith C, Emberson J, et al. Interpretation of the evidence for the efficacy and safety of statin therapy. Lancet 2016;388:2532-61.

31 van Driel ML, Morledge MD, Ulep R, et al. Interventions to improve adherence to lipid-lowering medication. Cochrane Database Syst Rev 2016;12:CD004371.

32 Gwadry-Sridhar FH, Manias E, Lal L, et al. Impact of interventions on medication adherence and blood pressure control in patients with essential hypertension: a systematic review by the ISPOR medication adherence and persistence special interest group. Value Health 2013;16:863-71.

33 National Health Service. Home blood pressure monitoring. England: National Health Service, 2020. https://www.england.nhs.uk/ourwork/ clinical-policy/cvd/home-blood-pressure-monitoring

34 NICE. Inclisiran for treating primary hypercholesterolaemia or mixed dyslipidaemia. National Institute for Health and Care Excellence, 2021. https://www.nice.org.uk/guidance/ta733 